\title{
Could aluminum be a new hidden allergen in type 1 hypersensitivity reactions when used as a drug additive?
}

\author{
Ali Kutlu', Ramazan Ucar², Ersin Aydin³, Sevket Arslan², Ahmet Zafer Calıskaner ${ }^{2}$ \\ ${ }^{1}$ Department of Allergy and Immunology, GATA Haydarpasa Training Hospital, Istanbul, Turkey \\ ${ }^{2}$ Department of Clinical Immunology and Allergy, Meram Faculty of Medicine, Necmettin Erbakan University, Konya, Turkey \\ ${ }^{3}$ Department of Dermatology, Kasimpasa Military Hospital, Istanbul, Turkey
}

Adv Dermatol Allergol 2016; XXXIII (3): 243-245

DOI: $10.5114 / a d a .2016 .60620$

Medications usually contain numerous additives and preservatives. Some of these agents have been reported as causative factors in adverse drug reactions, including asthma attacks, urticaria and/or angioedema, and even severe systemic anaphylaxis. Moreover, allergens such as additives and preservatives may be hidden as they are not easily identified during etiological investigations.

Aluminum is one of the most widely used adjuvants in medications and vaccines. It is also used as a coloring agent in the pharmaceutical and cosmetic industries. In the case presented here, we report on a patient who experienced anaphylaxis in response to a tetanus vaccination and urticaria attacks after administration of a colchicum preparation, which suggested that aluminum (used as an adjuvant in both drugs) could be a hidden allergen responsible for both reactions.

A 26-year-old female patient had been followed up at two allergy clinics because of the multidrug allergy syndrome and food allergies. The patient described allergic reactions (urticaria and angioedema) after use of a large number of non-steroidal anti-inflammatory drugs (NSAIDs) and antibiotics, most of which had been proven by provocation tests and specific lgE measurements. She was also allergic to inhalant allergens (grass pollens and latex), and many foods (including nuts, peanuts, soy beans, wheat flour, banana, kiwi, tomatoes, potatoes, and peaches, some of which are known to be cross-sensitive with latex).

Furthermore, she was reactive to numerous antihistamine preparations and methylprednisolone, as had been proven by provocation tests. In addition, at the age of 6 , she was diagnosed with Familial Mediterranean fever (FMF) due to recurrent abdominal pain and fever, which was confirmed by the presence of a genetic mutation. She is currently on a daily colchicum regimen. Colchicum preparation had been tolerated well by the patient for several years, but in recent years she has reported urticaria attacks. This reaction started after a change in the pharmaceutical form of her medication.

The patient was seen after an anaphylactic attack after a tetanus vaccine. She was admitted to the emergency department (ED) because of a contaminated sharp injury and a standard dose of tetanus vaccine was administered (Tetavax $0.5 \mathrm{ml}$, Sanofi Pasteur, by the intramuscular (IM) route). Approximately 10 min after the injection she developed widespread urticaria, shortness of breath, hoarseness, palpitations, and hypotension (according to the ED medical records). She was treated successfully with epinephrine $(0.3 \mathrm{mg}, \mathrm{IM})$ and no further therapeutic interventions were needed. The patient had undergone further etiological investigations as a result of the reaction described above.

The patient's medical history had been evaluated many times and all available allergy tests had been performed. In the last episode, she suffered a systemic reaction after a "safe" tetanus vaccine. There are several reports of systemic allergic reactions in children with a history of milk allergy after a tetanus vaccination that may contain trace amounts of casein (an allergenic milk protein) [1]. However, this patient has no history of a milk allergy.

The described anaphylactic reaction was moderate to severe. We investigated the excipients of the tetanus vaccine as there exist only limited and somewhat controversial data on anaphylactic reactions to tetanus toxoids. The tetanus toxoid was adsorbed to aluminum hydroxide $(0.6 \mathrm{mg})$ and the excipients were sodium chloride, disodium phosphate dihydrate, and monopotassium phosphate [2].

Address for correspondence: Ersin Aydin MD, Department of Dermatology, Kasimpasa Military Hospital, 34440 Istanbul, Turkey, phone: +90 5452884402, e-mail: drersinaydin@yahoo.com Received: 9.06.2015, accepted: 1.10.2015. 


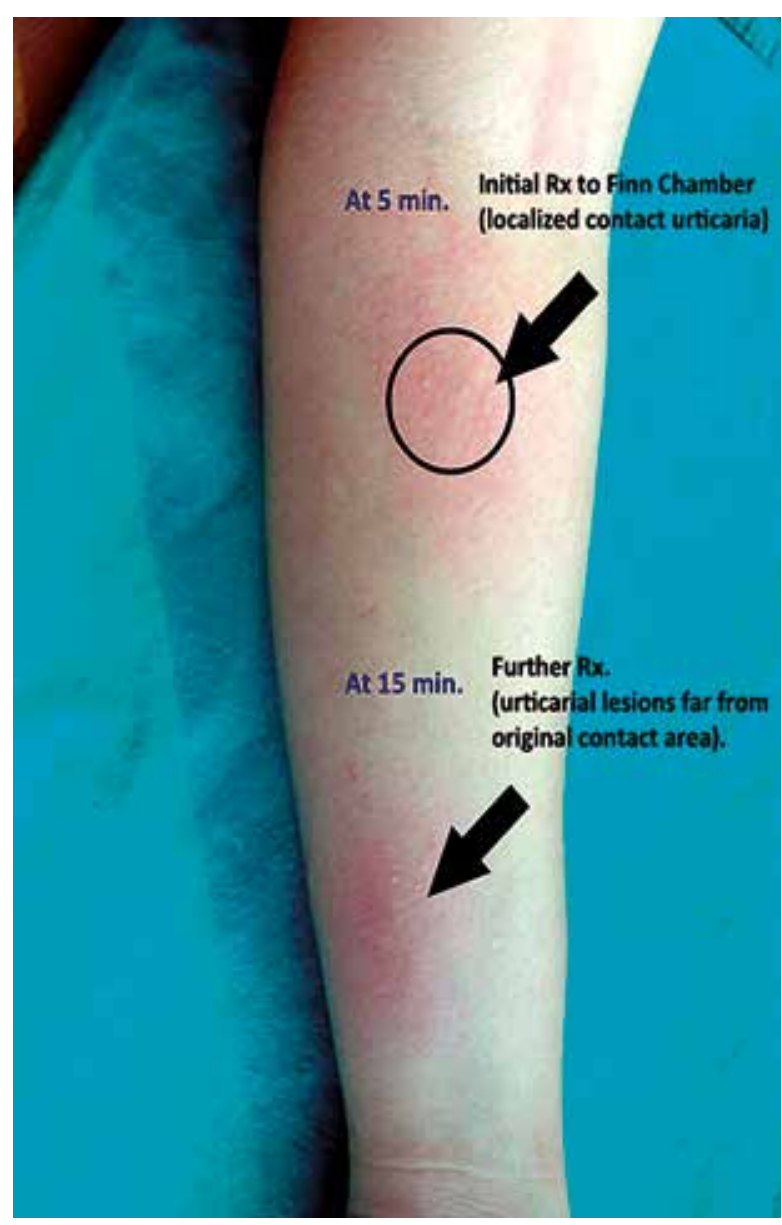

Figure 1. Initial and further urticarial lesions after contact with a Finn Chamber ${ }^{\circledR}$

The urticaria reaction to colchicum was also reevaluated during this process to identify a probable shared allergen between the vaccine and the colchicum. Colchicum induced urticaria only after a different pharmaceutical form of the drug had been taken. The excipients of the previous colchicum preparation (Colchicum-Dispert dragée, Recordati, Istanbul) were investigated and compared with the new form. The different excipient was aluminum, which was also present in the tetanus vaccine; a diagnostic evaluation of aluminum was carried out on the patient. We used a patch test chamber (Finn Chamber $^{\circledast}, \varnothing=8 \mathrm{~mm}$; Epitest Ltd Oy, Tuusula, Finland) produced from pure elemental aluminum as the allergen, since there was no commercially available, validated aluminum test material to investigate a type 1 reaction. One Finn Chamber ${ }^{\oplus}$ was placed directly onto the inner side of the forearm (without scratching). Histamine at 1\% was used as a positive control, $0.9 \%$ saline and $0.4 \%$ phenol solutions were used as negative controls (by the prick test method). Hyperemic urticarial lesions were observed under the Finn chamber ${ }^{\circledast}$ after 5 min, and after approxi- mately 10 min, urticarial lesions expanding up the entire arm were observed (Figure 1).

To confirm the diagnosis of aluminum allergy, an oral provocation test with the colchicum preparation used previously was performed, but the patient tolerated this form well and there were no signs of any urticarial lesions. Finally, the patient was diagnosed with a type 1 hypersensitivity reaction to aluminum.

Aluminum compounds are widely used in medicines as drug additives, and especially in vaccines and antigen extracts used in allergen-specific immunotherapy [3]. Aluminum is also present in toothpaste, dental cements, antiperspirants and pigments used in tattooing. Despite the extent of aluminum exposure, clinically relevant contact allergy to aluminum is not a common problem [4].

Hypersensitivity reactions to drugs are classified as allergic or non-allergic according to the immune system response. Multiple drug allergy syndrome (MDAS) is defined as an allergic reaction to two or more structurally unrelated drugs, and is a rare condition. The vast majority of patients with drug allergies are allergic to a single drug or a group of drugs [5].

The majority of reported adverse reactions to aluminum-adsorbed vaccines (alone or in the combination of DTaP, hepatitis B and human papilloma virus) are intensely itchy subcutaneous nodules (granulomas) at the injection site which can persist for months or even years $[6,7]$. Type-1 hypersensitivity to aluminum adsorbed to a vaccine has not been reported in the English-language literature.

Although the patient reported urticaria after using a new pharmaceutical form of colchicum, that reaction was not attributed to aluminum. The new form included several additives, including lactose monohydrate, starch, talcum powder, Kollidon ${ }^{\oplus}$ VA 64, magnesium stearate, stearic acid, titanium dioxide, polyvinyl alcohol, polyethylene glycol, FD\&C yellow \#6, aluminum lake, and Ponceau 4R. In addition, type-1 hypersensitivity to aluminum as a drug additive had not been reported. After the onset of anaphylaxis after the tetanus vaccine containing aluminum hydroxide, sodium chloride, disodium dihydrate phosphate and monopotassium phosphate, we considered aluminum to be the culprit agent.

Despite many additives being used in the drug industry, most have not been proven to be related to any hypersensitivity reactions, and real IgE-mediated reactions are rare. We performed a contact test with an empty Finn Chamber ${ }^{\circledR}$ made of elemental aluminum to confirm a type 1 allergic reaction to aluminum. The reaction (generalized urticaria) was compatible with a type 1 hypersensitivity reaction.

In conclusion, contact allergy to aluminum has been reported after numerous vaccinations and allergen-specific immunotherapy as intensely itchy subcutaneous granulomas, but rarely as occupational contact dermatitis. To our knowledge, this is the first reported case of 
anaphylaxis after a tetanus vaccination that includes aluminum as an adsorbent, in which the role of aluminum was confirmed by a contact test.

\section{Conflict of interest}

The authors declare no conflict of interest.

\section{References}

1. Kattan JD, Konstantinou GN, Cox AL, et al. Anaphylaxis to diphtheria, tetanus, and pertussis vaccines among children with cow's milk allergy. J Allergy Clin Immunol 2011; 128: 215-8.

2. Available at: http://drug.fda.moph.go.th/zone_search/ files/1C_22_54_B_Tetavax.Pdf, 2016.

3. Netterlid E, Hindsen M, Bjork J, et al. There is an association between contact allergy to aluminium and persistent subcutaneous nodules in children undergoing hyposensitization therapy. Contact Dermatitis 2009; 60: 41-9.

4. Gente Lidholm A, Bergfors E, Inerot A, et al. Unexpected loss of contact allergy to aluminium induced by vaccine. Contact Dermatitis 2013; 68: 286-92.

5. Macy E, Ho NJ. Multiple drug intolerance syndrome: prevalence, clinical characteristics, and management. Ann Allergy Asthma Immunol 2012; 108: 88-93.

6. Bergfors E, Trollfors B, Inerot A. Unexpectedly high incidence of persistent itching nodules and delayed hypersensitivity to aluminium in children after the use of adsorbed vaccines from a single manufacturer. Vaccine 2003; 22: 64-9.

7. Bergfors E, Trollfors B. Sixty-four children with persistent itching nodules and contact allergy to aluminium after vaccination with aluminium-adsorbed vaccines-prognosis and outcome after booster vaccination. Eur J Pediatr 2013; 172: 171-7. 